Pacific Journal of Mathematics

ON THE REPRESENTATION THEORY FOR CYLINDRIC 


\title{
ON THE REPRESENTATION THEORY FOR CYLINDRIC ALGEBRAS
}

\author{
DONALD MONK
}

The main purpose of this paper is to give some new sufficient conditions for the representability of infinite dimensional cylindric algebras. We also discuss certain problems and results in the representation theory reported on by Henkin and Tarski in [5].

In general we adopt the notation of [5]. $\S 1$ contains some additional notation, the statement of a representation theorem of Henkin and Tarski frequently used in this paper, and an embedding theorem which throws some light on that representation result. $\S 2$ is devoted mainly to some simple proofs for known results about the general algebraic theory of representable cylindric algebras. Then in $\S 3$ we turn to representation theory proper. The first result of this section gives a sufficient condition for representability in terms of isomorphic reducts of an algebra (this result was independently obtained by Alfred Tarski). Then follows the definition of a new class of cylindric algebras, diagonal cylindric algebras. The main theorem of this paper is that every diagonal cylindric algebra is representable; this result represents a considerable improvement of some previously known representation theorems. Several interesting corollaries are derived from this result.

1. Introduction. We use the notation of [5] with the following additions. For abbreviational purposes we use standard logical notation: $\rightarrow$ (implies), $\vee$ (there exists), and $\Lambda$ (for all). The identity map on a set $A$ is denoted by $\delta_{A}$. The function $f$ restricted to the subset $A$ of its domain is denoted by $f \nmid A$. If $R$ is a binary relation and $A$ is a set, then $R^{*}(A)=\left\{y \mid \mathbf{V}_{x \in A}\langle x y\rangle \in R\right\}$. If $\mathfrak{A}=\left\langle A,+, \cdot,-, c_{\kappa}, d_{\kappa \lambda}\right\rangle_{\kappa, \lambda<\infty}$ is a $C A_{\alpha}$, then $\mathfrak{A}_{0}=\langle A,+, \cdot,-\rangle$ is the Boolean part of $\mathfrak{A}$. Directed systems are understood in the sense of [7] p. 65.

We need some notions of general algebra, adapted from [9]. Let $\boldsymbol{K}$ be a class of similar algebras; say all algebras of $\boldsymbol{K}$ are indexed by a nonempty set $N_{\boldsymbol{K}}$, so that if $\mathfrak{A} \in \boldsymbol{K}$ then $\mathfrak{U}=\left\langle A, O_{i}\right\rangle_{i \in_{N} \boldsymbol{K}}$, the $O_{i}$ being operations on $A$. We let $\boldsymbol{H} \boldsymbol{K}=$ the class of all homomorphic images of algebras of $\boldsymbol{K}, \boldsymbol{P K}=$ the class of all Cartesian products of systems of

Received December 15, 1960. The results of this paper were obtained in part while the author was a National Science Foundation predoctoral fellow and in part while the author was engaged in a research project in the foundations of mathematics directed by Alfred Tarski and supported by the National Science Foundation (Grant No. G-14006) The author wishes to thank Professor Alfred Tarski for the valuable advice he gave during the preparation of this paper. The results of this paper constitute part of the author's doctoral dissertation submitted in May 1961 at the University of California, Berkeley. 
algebras of $\boldsymbol{K}$, and $\boldsymbol{S K}=$ the class of all subalgebras of algebras of $\boldsymbol{K}$. If $J \subseteq N_{\boldsymbol{K}}$ and $\mathfrak{A} \in \boldsymbol{K}$, we let $\mathfrak{A}_{J}=\left\langle A, O_{i}\right\rangle_{i \in J}$; and we let $\boldsymbol{K}_{J}=\left\{\mathfrak{U}_{J} \mid \mathfrak{A} \in \boldsymbol{K}\right\}$.

To fit cylindric algebras into this scheme of universal algebra, let us make the following agreement. For each ordinal $\alpha$, let $M_{\alpha}=$ $\{0,1,2,\langle 0, \kappa\rangle,\langle 0, \kappa, \lambda\rangle\}_{\kappa, \lambda<\alpha}$. If $\mathfrak{A}=\left\langle A,+, \cdot,-, c_{\kappa}, d_{\kappa \lambda}\right\rangle_{\kappa, \lambda<\alpha}$ is a $C A_{\alpha}$, we let $O_{0}=+, O_{1}=\cdot, O_{2}=-, O_{\langle 0, \kappa\rangle}=c_{\kappa}$, and $O_{\langle 0, \kappa, \lambda\rangle}=d_{\kappa \lambda}$ for all $\kappa, \lambda<\alpha$; finally we let $\mathfrak{U}^{*}=\left\langle A, O_{i}\right\rangle_{i \in_{M_{n}}}$. We let $C A_{\alpha}^{*}=\left\{\mathfrak{I}^{*} \mid \mathfrak{A} \in C A_{\alpha}\right\}$. Thus $C A_{\alpha}^{*}$ is a class of similar algebras in the above sense. When no confusion results we shall identify $C A_{\alpha}$ with $C A_{\alpha}^{*}$.

In several of the proofs below we use a method of construction whose general form is as follows. We are given a class $\boldsymbol{K}$ of similar algebras, a directed system $\mathfrak{D}=\langle D, \geqq\rangle$, and, for each $d \in D$, an element $\mathfrak{A}_{a}$ of $\boldsymbol{K}$. We let $R=\left\{\langle f, g\rangle \mid f, g \in \Pi_{a \in_{D}} A_{d}\right.$ and $\bigvee_{a \in D} \Lambda_{e} \in_{D}\left(d \leqq e \rightarrow f_{e}=\right.$ $\left.\left.g_{e}\right)\right\}$. Clearly $R$ is a congruence relation on $\Pi_{a \in D} \mathfrak{A}_{d} ; R$ is called the eventually equal congruence of $\mathfrak{A}$ and $\mathfrak{D} .^{1}$ In case $\boldsymbol{K}=C A_{\alpha}^{*}$ for some $\alpha,\{f \mid\langle f, 0\rangle \in R\}$ is called the eventually zero ideal of $\mathfrak{A}$ and $\mathfrak{D}$. In case $J \subseteq N_{K}$ and $\mathfrak{B}$ is a subalgebra of $\mathfrak{A}_{d J}$ for each $d \in D$, we may define natural isomorphisms $g$ and $f$ of $\mathfrak{B}$ into $\prod_{a \in D} \mathfrak{A}_{d J}$ and $\left[\prod_{a \in D} \mathfrak{A}_{d} / R\right]_{J}$ respectively. For each $b \in B$ and $d \in D$ let $g(b)_{d}=b$. For each $b \in B$ let $f(b)=[g(b)]$. If $\boldsymbol{K}=C A_{\alpha}$ and $J=\{0,1,2\}, g$ and $f$ are called the natural Boolean isomorphisms of $\mathfrak{B}$ into $\prod_{a \in D} \mathfrak{A}_{a}$ and $\prod_{a \in D} \mathfrak{A}_{a} / R$ respectively.

The essential steps in the proofs of the representation theorems here presented use the following theorem of Henkin and Tarski (see [5] Theorem 2.15).

THEOREM A. $A C A_{a} \mathfrak{A}$ is representable if and only if for each $\kappa<\omega \mathfrak{A}$ can be neatly embedded in some $C A_{\alpha+\kappa}$.

There now exist purely algebraic proofs of this theorem. Theorem $A$ is to be contrasted with the following theorem:

THEOREM 1. If $\delta \geqq \alpha \geqq \omega$, then every $C A_{\alpha}$ is embeddable in some $C A_{\delta}$, i.e., is a subalgebra of the $\alpha$-reduct of some $C A_{\delta}{ }^{2}$

Proof. It suffices to take the case $\delta=\alpha+1$. For each $\beta<\omega$ we define $\gamma^{(\beta)}$ with domain $\alpha+1$ by:

$$
\gamma_{\kappa}^{(\beta)}= \begin{cases}\kappa & \text { if } \kappa<\beta, \\ \kappa+1 & \text { if } \beta \leqq \kappa<\omega, \\ \kappa & \text { if } \omega \leqq \kappa<\alpha, \\ \beta & \text { if } \kappa=\alpha,\end{cases}
$$

${ }^{1} \Pi_{d \in D} \mathscr{P}_{d} / R$ is a reduced product in the sense of Frayne, Scott, and Tarski (Notices Amer. Math. Soc., 5 (1958) 673). In fact, let $J=\left\{X \mid X \subseteq D\right.$ and $\left.\vee_{a \in D} \wedge_{e \in D}(d \leqq e \rightarrow e \in X)\right\}$. Then $J$ is an ideal in the field of all subsets of $D$, and $R$ is the congruence relation on $\Pi_{a \in D} \mathscr{A}_{a}$ determined by $J$.

2 This theorem, due to the author, is stated in [5]. 
for all $\kappa<\alpha+1$. Thus $\gamma^{(\beta)}$ is one-to-one. Let $\mathfrak{A}$ be a given $C A_{\alpha}$, and let $\mathfrak{B}_{\beta}$ be the $\alpha+1, \gamma^{(\beta)}$-reduct of $\mathfrak{A}$. Let $I$ be the eventually zero ideal of $\mathfrak{B}$ and $\langle\omega, \geqq\rangle$. Let $\mathfrak{F}=\prod_{\beta<\omega} \mathfrak{B}_{\beta} / I$, and let $g, f$ be the natural Boolean isomorphisms of $\mathfrak{A}$ into $\Pi_{\beta>\omega} \mathfrak{B}_{\beta}$ and $\sqrt{5}$ respectively. If $0 \leqq \kappa<\beta<\omega$ or $0 \leqq \beta<\omega \leqq \kappa<\alpha$, then $\gamma_{\kappa}^{(\beta)}=\kappa$, and so, with $a \in A$, $\left(c_{\kappa} g(a)\right)_{\beta}=$ $c_{\kappa}^{\mathfrak{B} \beta} g(a)_{\beta}=c_{\gamma_{\kappa}^{(\beta)}}^{\mathfrak{A}} a=c_{\kappa}^{\mathfrak{Y}} a=g\left(c_{\kappa} a\right)_{\beta}$; similarly for diagonal elements. It follows that $f$ is a cylindric isomorphism of $\mathfrak{A}$ into the $\alpha$-reduct of $\mathfrak{F}$, as required..$^{3}$

Since for each $\alpha \geqq \omega$ there are non-representable $C A_{\alpha}$ 's, Theorems $A$ and 1 indicate the significance of the notion of neat embedding.

2. Universal algebra and cylindric algebra. In [5], Henkin and Tarski state several universal algebraic properties of representable cylindric algebras, indicating that their proofs use in an essential way some metamathematical results. Thus after proving that $R C A_{\alpha}$ is a universal class, they infer that

(i) a cylindric algebra is representable if and only if every finitely generated subalgebra of it is representable, and

(ii) a cylindric algebra is representable if and only if every finite reduct of it is representable.

Further, after proving that $R C A_{\alpha}$ is equational they infer that $R C A_{\alpha}$ is closed under the taking of homomorphic images. For all these algebraic results they raise the question concerning the existence of simple algebraic (as opposed to metamathematical) proofs.

With the essential help of Theorem A, which, as mentioned above, has algebraic proofs, we shall give algebraic proofs of the above results. In addition, we obtain a new proof of the equational character of $R C A_{\alpha}$.

\section{TheOREM 2. A homomorphic image of an $R C A_{\alpha}$ is an $R C A_{\alpha}{ }^{4}$}

Proof. Suppose $\mathfrak{A}$ is an $R C A_{\alpha}$ and $I$ is a cylindric ideal in $\mathfrak{A}$; we want to show that $\mathfrak{A} / I$ is an $R C A_{\alpha}$. Let $\mathfrak{B}$ be a $C A_{\alpha+\kappa}$ such that $\mathfrak{A}$ is neatly embedded in $\mathfrak{B}$ (by Theorem $A$ ), where $\kappa<\omega$. Let $J$ be the ideal in $\mathfrak{B}$ generated by $I$. Clearly $J=\left\{b \mid b \in B\right.$ and $\left.\mathbf{V}_{a \in I}(b \leqq a)\right\}$, and so $J \cap A=I$. It follows that the natural Boolean homomorphism of $\mathfrak{A} / I$ into $\mathfrak{B} / J$ is a cylindric isomorphism of $\mathfrak{A} / I$ onto an algebra neatly embedded in $\mathfrak{B} / J$, and by Theorem $\mathrm{A}$ our theorem follows.

It is easy to see that $R C A_{\alpha}$ is closed under direct products and subalgebras. Hence by Birkhoff's theorem (Theorem 2.1 of [9]), $R C A_{\alpha}$ is equational. Thus in particular, $R C A_{\alpha}$ is a universal class, and the above characterizations (i) and (ii) of $R C A_{\alpha}$ follow. Recently the author

${ }^{3}$ Theorem 1 can also be easily proved metamathematically. In fact, it was such a proof that first occurred to the author.

4 [5], Theorem 2.20 . 
obtained simple algebraic proofs of these two characterizations. Alfred Tarski, upon being informed of these proofs, recalled that in 1955 Saunders MacLane outlined to him a proof of a universal algebraic theorem from which (i) follows; the author's proof of (i) was a specialization of MacLane's proof. Since MacLane's proof has never appeared in print, we shall take this opportunity to present it here. Subsequent to the above work, the author obtained a corresponding algebraic proof of a generalization of (ii).

Of the two corollaries below, the first is a strict specialization of the universal algebraic case, while for the second corollary we apply an additional argument.

THEOREM 3. Let $\boldsymbol{K}$ be a class of similar algebras such that $\boldsymbol{H K}=$ $\boldsymbol{K}, \boldsymbol{P K}=\boldsymbol{K}$, and $\boldsymbol{S} \boldsymbol{K}=\boldsymbol{K}$. Then for every algebra $\mathfrak{A}, \mathfrak{A} \in \boldsymbol{K}$ if (and only if) every finitely generated subalgebra of $\mathfrak{A}$ is in $\boldsymbol{K}$.

Proof. The necessity of the condition is obvious. Now suppose that every finitely generated subalgebra of $\mathfrak{A}$ is in $\boldsymbol{K}$. Let $I=\{F \mid F$ is a finite subset of $A\}$, and for each $F \in I$ let $\mathfrak{B}_{F}$ be the subalgebra of $\mathfrak{A}$ generated by $F$. Let $R$ be the eventually equal congruence of $\mathfrak{B}$ and

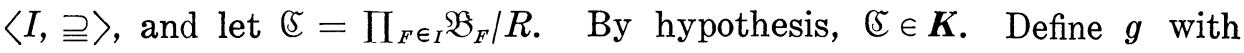
domain $A$ and range included in $\prod_{F \in I} B_{F}$ by:

$$
g(a)_{F}=\left\{\begin{array}{l}
\text { any element of } B_{F} \text { if } a \notin B_{F}, \\
a \text { if } a \in B_{F},
\end{array}\right.
$$

for all $a \in A$ and $F \in I$. It is easy to see that the function $f$, defined by $f(a)=[g(a)]$ for all $a \in A$, is an isomorphism of $\mathfrak{A}$ into $\sqrt{5}$. Hence $\mathfrak{A} \in \boldsymbol{K}$.

From Theorems 2 and 3 we obtain:

CoRollaRy. $\mathfrak{A} \in R C A_{\alpha}$ if (and only if) every finitely generated subalgebra of $\mathfrak{A}$ is representable. ${ }^{5}$

THEOREM 4. Let $\boldsymbol{K}$ be a class of similar algebras such that $\boldsymbol{H K}=$ $\boldsymbol{K}, \boldsymbol{P K}=\boldsymbol{K}$, and $\boldsymbol{S K}=\boldsymbol{K}$. Then $\mathfrak{A} \in \boldsymbol{K}$ if (and only if) for every finite subset $F$ of $N_{\boldsymbol{K}}$ we have $\mathfrak{A}_{F} \in \boldsymbol{S} \boldsymbol{K}_{F}$.

Proof. The necessity is obvious. Now suppose that the above condition holds. For each finite subset $F$ of $N_{\boldsymbol{K}}$ choose $\mathfrak{B}^{(F)} \in \boldsymbol{K}$ such that $\mathfrak{A}_{F} \subseteq \mathfrak{B}_{F^{(F)}}$. Choose $i_{0} \in N_{K}$. Let $I=\left\{F \mid F\right.$ is a finite subset of $N_{\boldsymbol{K}}$ and $\left.i_{0} \in F\right\}$. Let $R$ be the eventually equal congruence of $\mathfrak{B}$ and $\langle I, \supseteq\rangle$, and let $\mathfrak{C}=\prod_{F \in I} \mathfrak{B}^{(F)} / R$. Let $g$ and $f$ be the natural isomorphisms of

${ }^{5}$ [5], Theorem 2.13 (i). 
$\mathfrak{H}_{\left\{i_{0}\right\}}$ into $\prod_{F \in I} \mathfrak{B}_{\left\{i_{0}\right\}}^{(F)}$ and $\mathfrak{E}_{\left\{i_{0}\right\}}$ respectively. We claim that $f$ is an isomorphism of $\mathfrak{A}$ into $\mathfrak{E}$. For, if $i \in N_{K}$, say with $O_{i}^{\mathfrak{Q}}$ binary, and if $a, b \in A$, we have for $\{i\} \subseteq F \in I$ :

$$
\begin{aligned}
{\left[g\left(O_{i}^{\mathfrak{R}}(a, b)\right)\right]_{F} } & =O_{i}^{\mathfrak{R}}(a, b) \\
& =O_{i}^{\mathfrak{Q} F}(a, b) \\
& =O_{i}^{\mathfrak{B}_{F}^{(F)}}(a, b) \\
& =O_{i}^{\mathfrak{B}^{(F)}}\left([g(a)]_{F},[g(b)]_{F}\right) \\
& =\left[O_{i}(g(a), g(b))\right]_{F} .
\end{aligned}
$$

Thus $f\left(O_{i}^{\mathscr{P}}(a, b)\right)=O_{i}(f(a), f(b))$. We deduce that $\mathfrak{A} \in \boldsymbol{K}$ by the hypothesis of the theorem.

Again, we have a corollary for cylindric algebras. As mentined previously, this corollary is not quite as immediate as the corollary to Theorem 3; we need the following lemma in order to derive the corollary easily.

Lemma 1. Let $\boldsymbol{K}$ be a class of similar algebras such that $\boldsymbol{P} \boldsymbol{K}=\boldsymbol{K}$ and $\boldsymbol{S K}=\boldsymbol{K}$. Suppose that $\mathfrak{A}$ is an algebra such that for all $x, y \in A$ with $x \neq y$ there is a homomorphism $f$ of $\mathfrak{A}$ onto an algebra $\mathfrak{B} \in \boldsymbol{K}$ such that $f(x) \neq f(y)$. Then $\mathfrak{A} \in \boldsymbol{K}$.

In case additionally $\boldsymbol{K}=C A_{\alpha}$ it is enough to assume that for all $x \in A$ with $x \neq 0$ there is a homomorphism $f$ of $\mathfrak{A}$ into an algebra $\mathfrak{B} \in \boldsymbol{K}$ such that $f(x) \neq 0$.

The proof of this lemma is simple; it is essentially due to Birkhoff ([1]).

The proof of necessity in the following corollary gives a simple proof of Theorem 2.12 of [5].

Corollary. $\mathfrak{U} \in R C A_{\alpha}$ if and only if every finite reduct of $\mathfrak{A}$ is representable. ${ }^{6}$

Proof. Necessity. Suppose $\mathfrak{A} \in R C A_{\alpha}$, i.e., $\mathfrak{A}$ is isomorphic to a sub-direct product of $C S A_{\alpha}$ 's. Now a reduct of a product of $C A_{\alpha}$ 's is equal to the product of the corresponding reducts. Hence we may assume that $\mathfrak{A}$ is a $C S A_{\alpha}$, say with base $U$. Suppose $\kappa<\omega$ and $\theta \in \alpha^{\kappa}$ is one-to-one; let $\mathfrak{B}$ be the $\kappa, \theta$-reduct of $\mathfrak{A}$. Suppose $b \in B$ and $b \neq \mathbf{0}$; choose $f \in b$. For each $g \in U^{\kappa}$ we define $g^{*} \in U^{\alpha}$ by:

$$
g_{\lambda}^{*}=\left\{\begin{array}{l}
g_{\theta^{-1} \lambda} \text { if } \lambda \in \text { range } \theta \\
f_{\lambda} \text { otherwise }
\end{array}\right.
$$

${ }^{6}[\mathbf{5}]$, Theorems $2.12,2.13$ (ii). 
Define $F(x)=\left\{g \in U^{\kappa} \mid g^{*} \in x\right\}$ for each $x \in B$. It is easy to verify that $F$ is a homomorphism of $\mathfrak{B}$ onto a $C S A_{\kappa}$ such that $F(b) \neq 0$. Since $b$ is arbitrary, we deduce from Lemma 1 that $\mathfrak{B} \in R C A_{\kappa}$.

Sufficiency. We now assume that every finite reduct of $\mathfrak{A}$ is representable. Let a finite subset $F$ of $M_{\alpha}$ be called regular if there is a finite subset $G$ of $\alpha$ such that $F=\{0,1,2,\langle 0, \kappa\rangle,\langle 0, \kappa, \lambda\rangle\}_{\kappa, \lambda \in G}$. Now it is known, and easy to see, that an $R C A_{\alpha}$ can be neatly embedded in an $R C A_{\beta}$ for each $\beta \geqq \alpha$; if we apply this argument here we see that, by our assumption, $\mathfrak{A}_{F}^{*} \subseteq \mathfrak{B}_{F}^{(F) *}$ for some $\mathfrak{B}^{(F)} \in R C A_{\alpha}$, for each regular finite subset $F$ of $M_{\alpha}$. If $F$ is any finite subset of $M_{\alpha}$, then there is a regular finite subset $G$ such that $F \cong G$, and so $\mathfrak{A}_{F}^{*}=\left(\mathfrak{U}_{G}^{*}\right)_{F} \subseteq\left(\mathfrak{B}_{G}^{(F) *}\right)_{F} \in$ $\left[\left(R C A_{\alpha}^{*}\right)_{G}\right]_{F}=\left(R C A_{\alpha}^{*}\right)_{F}$. Hence by Theorem 4, $\mathfrak{A}^{*} \in R C A_{\alpha}^{*}$, i.e., $\mathfrak{A} \in R C A_{\alpha}$.

We conclude this section with the following theorem.

Theorem 2'. Let $\alpha$ and $\kappa$ be ordinals. Let $\boldsymbol{K}$ be the class of all $C A_{\alpha}$ 's which can be neatly embedded in a $C A_{\alpha+\kappa}$. Then $\boldsymbol{K}$ is an equational class.

Proof. Clearly $\boldsymbol{K}$ is closed under direct products and subalgebras. The proof of Theorem 2 may be applied to show that $\boldsymbol{K}$ is closed under homomorphisms. Our theorem is now a consequence of Birkhoff's theorem.

From this theorem we can derive two corollaries similar to the above stated corollaries. This can be done metamathematically, in the obvious way, or mathematically as follows. For the first corollary we can again use Theorem 3, while for the second we can use a direct argument similar to the proof of Theorem 4. (We do not know of any way of using Theorem 4 or something like it to derive the second corollary.)

3. Some representation theorems. Now we shall prove several new sufficient conditions for the representability of cylindric algebras. The following simple lemma will be found useful in the proofs of the main results.

Lemma 2. Let $\alpha, \beta$, and $\gamma$ be ordinals, and suppose that $\tau \in \beta^{a+\gamma}$ is one-to-one. Suppose $\mathfrak{A}$ is a $C A_{\alpha}, \mathfrak{B}$ is a $C A_{\beta}, T \in B^{A}$, and the following conditions hold:

(i) $T$ is a Boolean homomorphism of $\mathfrak{A}$ into $\mathfrak{B}$,

(ii) $c_{\tau \kappa}^{\mathfrak{B}} \circ T=T \circ c_{\kappa}^{\mathfrak{A}}$ for all $\kappa<\alpha$,

(iii) $T\left(d_{\kappa \lambda}^{\mathfrak{Q}}\right)=d_{\tau \kappa, \tau \lambda}^{\mathfrak{B}}$ for all $\kappa, \lambda<\alpha$.

Then $T$ is a cylindric homomorphism of $\mathfrak{A}$ into the $\alpha$-reduct of some $C A_{\alpha+\cdots}$. If in addition the following condition holds:

(iv) $c_{\tau \kappa}^{\mathfrak{B} \circ T}=T$ for $\alpha \leqq \kappa<\alpha+\gamma$, 
then $T$ is a cylindric homomorphism of 2 into an algebra neatly embedded in some $C A_{\alpha+}$.

Proof. Let $\sqrt{S}$ be the $\alpha+\gamma, \tau$-reduct of $\mathfrak{B}$, and let $\mathfrak{D}$ be the $\alpha$-reduct of $\sqrt{5}$. Then $T$ is a cylindric homomorphism of $\mathfrak{A}$ into $\mathfrak{D}$, for $T \circ c_{k}^{\mathfrak{A}}=c_{\tau \kappa}^{\mathfrak{Q}} \circ T$ (by (ii)) $=c_{\kappa}^{\mathfrak{E}} \circ T=c_{k}^{\mathfrak{D}} \circ T$ for each $\kappa<\alpha$, and $T\left(d_{\kappa \lambda}^{\mathfrak{A}}\right)=$ $d_{\tau \kappa, \tau \lambda}^{\mathfrak{B}}=d_{\kappa \lambda}^{\mathbb{S}}=d_{\kappa \lambda}^{\mathbb{D}}$ for all $\kappa, \lambda<\alpha$. If in addition (iv) holds, then for $\alpha \leqq \kappa<\alpha+\gamma$ we have $c_{\kappa}^{\sqrt{\varsigma}} \circ T=c_{\tau \kappa}^{\mathfrak{B}} \circ T=T$.

As a consequence of Theorem $\mathrm{A}$ and Lemma 2 we have the following representation theorem, which was independently obtained by Alfred Tarski.

THEOREM 5. Assume that $\mathfrak{A}$ is a $C A_{\alpha}, \sigma$ is a one-to-one element of $\alpha^{\alpha}$ such that $\alpha \sim$ range $(\sigma)$ is infinite, and $\mathfrak{B}$ is the $\alpha, \sigma$-reduct of $\mathfrak{A}$. Suppose $T$ is an isomorphism of $\mathfrak{A}$ into $\mathfrak{B}$ such that $c_{\kappa}^{\mathfrak{A}} T(x)=T(x)$ whenever $x \in A$ and $\kappa \in \alpha \sim$ range $(\sigma)$. Then $\mathfrak{A}$ is representable.

Proof. Let $\tau$ be a one-to-one element of $\alpha^{\alpha+\omega}$ such that $\tau \mid \alpha=\sigma$.

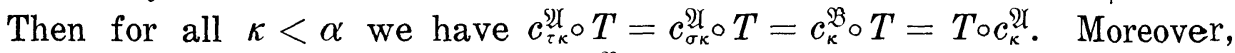
for all $\kappa, \lambda<\alpha$ we have $T\left(d_{\kappa \lambda}^{\mathfrak{Y}}\right)=d_{\kappa \lambda}^{\mathfrak{B}}=d_{\tau \kappa, \tau \lambda}^{\mathscr{Y}}$. Finally, if $\alpha \leqq \kappa<\alpha+\omega$, then $c_{\tau \kappa}^{\mathfrak{A}} \circ T=T$. Hence by Lemma $2 \mathfrak{U}$ can be neatly embedded in a $C A_{\alpha+\omega}$, and our theorem follows from Theorem A.

We should mention that recently Tarski obtained a stronger version of Theorem 5, in which the condition " $\alpha \sim$ range $(\sigma)$ is infinite" is replaced by the condition " $\alpha \sim$ range $(\sigma) \neq 0$ ".

Theorem 5 leads to an interesting insight into the relationship between cylindric and polyadic algebras, of a different kind from the insight obtained from the relationships established in [2]. A polyadic algebra with equality is, roughly speaking, a cylindric algebra with two additional structures: infinite cylindrification, and substitution (see [3]). If we eliminate only the infinite cylindrification, we arrive at a notion of a substitution on a cylindric algebra. A substitution on a $C A_{\alpha} \mathfrak{A}$ is a function $S \in\left(A^{A}\right)^{\alpha^{\alpha}}$ which satisfies certain natural conditions (due to Halmos). As a corollary of Theorem 5 we easily see that if $\mathfrak{A}$ is a $C A_{a}$ with a substitution and if $\alpha \geqq \omega$, then $A$ is representable. Now from [6] it is known that every infinite dimensional polyadic algebra is representable, while there are infinite dimensional polyadic equality algebras which are not representable (with equality corresponding to the functional equality). Here by representable we mean as in cylindric algebras-isomorphic to a subdirect product of $\bigcirc$-valued functional polyadic algebras. Our corollary shows that by eliminating infinite cylindrification we recapture representation.

It is natural to ask if the corollary can be strengthened by replacing "substitution" by "finite substitution"-a concept defined like 
that of substitution, but in which $S$ applies only to those $\tau \in \alpha^{\alpha}$ for which there is a finite subset $F$ of $\alpha$ such that $\tau \mid \alpha \sim F=\delta_{\alpha \sim F}$. The answer is no: for each $\alpha \geqq \omega$ there exists a $C A_{\alpha}$ with a finite substitution which is not representable. The construction of such algebras depends on the results of [8], which in turn depend upon unpublished work of Henkin and Tarski.

We now define a class of cylindric algebras which includes both the class of simple infinite dimensional cylindric algebras and the class of dimensionally complemented cylindric algebras. A $C A_{\alpha} \mathfrak{A}$ is called a diagonal cylindric algebra $\left(G C A_{\alpha}\right)$ provided that for every non-zero $a \in A$ and every finite subset $F$ of $\alpha$ there are distinct $\kappa, \lambda \in \alpha \sim F$ such that $a \cdot d_{\kappa \lambda} \neq 0$. The importance of this concept derives from the following theorem:

THEOREM 6. Every diagonal cylindric algebra is representable.?

Proof. Let $\mathfrak{A}$ be a $G C A_{\alpha}$. We want to apply Lemma 1, with $\boldsymbol{K}$ replaced by the class of all $C A_{\alpha}$ 's which can be neatly embedded in $C A_{\alpha+1}$ 's. Hence suppose that $a \in A$ and $a \neq 0$. Since $\mathfrak{A} \in G C A_{\alpha}$ we can define functions $\mu, \nu$ with domain $\omega$ inductively by letting $\mu_{\kappa}$ and $\nu_{\kappa}$ be distinct members of $\alpha \sim\left\{\mu_{\lambda}, \nu_{\lambda} \mid \lambda<\kappa\right\}$ such that $a \cdot d_{\mu_{\kappa} \nu_{\kappa}} \neq 0$.

Now we prepare to apply Lemma 2. It is easy to see that there is a unique $\tau \in \alpha^{\alpha+1}$ such that the following conditions hold:

(1) $\tau$ is one-to-one,

(2) $\tau$ is the identity on $\alpha \sim\left\{\mu_{\kappa}, \nu_{\kappa} \mid \kappa<\omega\right\}$,

(3) $\tau \mu_{\kappa}=\nu_{\kappa}$ for each $\kappa<\omega$,

(4) $\tau \nu_{\kappa}=\mu_{\kappa+1}$ for each $\kappa<\omega$,

(5) $\tau \alpha=\mu_{0}$.

For each $\kappa<\omega$, let $\mathfrak{B}_{\kappa}=\mathfrak{A}$. Let $I$ be the eventually zero ideal of $\mathfrak{B}$ and $\left\langle\omega\right.$, $\geqq$, and let $\mathfrak{E}=\mathfrak{A}^{\omega} / I$. For each $x \in A$ and $\kappa<\omega$, define

$$
f(x)_{\kappa}=S_{\nu_{0}}^{\mu_{0}} S_{\mu_{1}}^{\nu_{0}} \cdots S_{\mu_{\kappa}-1}^{\nu_{\kappa}} S_{\nu_{\kappa}}^{\mu_{\kappa}} x,
$$

where $S_{\rho}^{\theta} x=c_{\theta}\left(d_{\theta \rho} \cdot x\right)$ for all $\theta, \rho<\alpha$ and $x \in A$. Let $T(x)=[f(x)]$ for all $x \in A$. The following statements may now be verified:

(6) $T$ is a Boolean homomorphism of $\mathfrak{A}$ into $C$,

(7) $c_{\tau \lambda}^{\mathfrak{E}} \circ T=T \circ c_{\lambda}^{\mathfrak{N}}$ for all $\lambda<\alpha$,

7 After reading a preliminary draft of this paper, Henkin obtained a generalization of this theorem, which may be stated as follows. If for every nonzero $x \in A$ and for every finite $\Gamma \leqq \alpha$ there is a $\xi \in \alpha \sim \Gamma$ and an endomorphism $T$ of $\mathfrak{X}_{0}$ such that $c_{\xi} \circ T=T, c_{\kappa} \circ T=T \circ c_{\kappa}$ for each $\kappa \in \Gamma$, and $T(x) \neq 0$, then $\mathfrak{A}$ is representable. 
(8) $T\left(d_{\lambda \mu}^{\mathfrak{A}}\right)=d_{\tau \lambda, \tau \mu}^{\mathfrak{S}}$ for all $\lambda, \mu<\alpha$,

(9) $c_{\tau \alpha}^{\mathfrak{S}} \circ T=T$.

In verifying (7), one can make use of the following easily verified arithmetic law:

(10) $S_{\theta}^{\rho} c_{\rho} S_{\pi}^{\theta} x=c_{\theta} S_{\theta}^{\rho} S_{\pi}^{\theta} x$ for all $x \in A$ and all distinct $\rho, \theta, \pi<\alpha$.

We can now apply Lemma 2 , and infer that $T$ is a cylindric homomorphism of $\mathfrak{A}$ into an algebra neatly embedded in a $C A_{\alpha+1}$. Suppose $T(a)=0$. Choose $k<\omega$ such that $f(a)_{\kappa}=0$. Applying successively $S_{\mu_{0}}^{\nu_{0}}, S_{\nu_{0}}^{\mu_{1}}, \cdots, S_{\nu_{\kappa}-1}^{\mu_{\kappa}}$ we infer that $S_{\nu_{\kappa}}^{\mu_{\kappa}} a=0$, and so $a \cdot d_{\mu_{\kappa} \nu_{\kappa}}=0$, which is a contradiction. Since $a$ is arbitrary, from Lemma 1 we conclude that $\mathfrak{A}$ can be neatly embedded in some $C A_{\alpha+1} \mathfrak{D}$. Let $g$ be an isomorphism of $\mathfrak{A}$ onto an algebra neatly embedded in $\mathfrak{D}$.

Let $N$ be maximal among ideals $P$ such that $g^{*}(A) \cap P=\{0\}$ (by Zorn's lemma). Let $\mathbb{F}=\mathfrak{D} / N$, and let $p r$ be the natural homomorphism of $\mathfrak{D}$ onto $\mathfrak{F}$. Clearly $p r \circ g$ is an isomorphism of $\mathfrak{A}$ onto an algebra neatly embedded in $\tilde{\xi}$. Suppose $x \in D, F$ is a finite subset of $\alpha$, and $[x] \leqq\left[-d_{\kappa \lambda}\right]$ for all distinct $k, \lambda \in \alpha \sim F$. Suppose that $x \notin N$. Then $N \cup\{x\}$ generates an ideal $P$ such that $P \cap g^{*}(A) \neq\{0\}$. Choose $y \in A$ such that $g(y) \neq 0$ and $g(y) \in P$. Then there are $\kappa_{0} . \cdots, \kappa_{\nu-1} \in \alpha+1$ and $n \in N$ such that $g(y) \leqq n+c_{\kappa_{0}} \cdots c_{\kappa_{\nu-1}} x$. Let $F^{\prime}=F \cup\left\{\kappa_{0}, \cdots, k_{\nu-1}\right\}$. Then $[g(y)] \leqq\left[-d_{\kappa \lambda}\right]$ for all distinct $\kappa, \lambda \in \alpha \sim F^{\prime}$; but this contradicts the fact that $\mathfrak{A}$ is a diagonal cylindric algebra.

It follows that $\&$ is a $G C A_{\alpha+1}$. Hence all the preceding proof can be applied inductively to give, in virtue of Theorem $A$, the desired result.

We now proceed to derive some consequences of Theorem 6 .

THEOREM 7. Every simple infinite dimensional algebra is a diagonal cylindric algebra, and so is representable.

Proof. Suppose $\mathfrak{A}$ is a simple $C A_{\alpha}, \alpha \geqq \omega, a \in A, a \neq 0$, and $F$ is a finite subset of $\alpha$. There are $\lambda \in \omega \sim 1$ and $\mu \in \alpha^{\lambda}$ such that $c_{\mu_{0}} \cdots c_{\mu_{\lambda-1}} a=$ 1. Choose $\kappa, \nu$ distinct in $\alpha \sim\left(F \cup\left\{\mu_{0}, \cdots, \mu_{\lambda-1}\right\}\right)$. If $a \cdot d_{\kappa \nu}=0$, then, applying $c_{\mu_{0}} \cdots c_{\mu_{\lambda-1}}$, we see that $d_{\kappa \nu}=0$; hence $0=1$, contradicting the simplicity of $\mathfrak{A}$.

From Theorem 7 we can infer the following negative theorem which limits the possible extensions of Theorem 1 .

Theorem 8. If $1<\alpha<\omega$, then it is not the case that every $C A_{a}$ can be embedded (in the sense of Theorem 1) in a $C A_{\omega}$.

Proof. Assume the contrary. Henkin and Tarski have constructed 
a non-representable $C A_{\alpha} \mathfrak{A}$, in unpublished work. Clearly we may assume that $\mathfrak{A}$ is simple. Let $\mathfrak{B}$ be a $C A_{\omega}$ such that $\mathfrak{A}$ is a subalgebra of the $\alpha$-reduct of $\mathfrak{B}$. Let $I$ be a maximal ideal in $\mathfrak{B}$, and let $\mathfrak{C}=\mathfrak{B} / I$. By Theorem 7, $\sqrt{5}$ is representable. Since $\mathfrak{U}$ is simple, $A \cap I=\{0\}$, and so the natural homomorphism of $\mathfrak{A}$ into $\mathfrak{S}$ is an isomorphism. It follows that $\mathfrak{A}$ is representable; but this is a contradiction.

A $C A_{\alpha} \mathfrak{A}$ is weakly dimensionally complemented, $\mathfrak{A} \in W D C A_{\alpha}$ if $\alpha \sim \Delta x$ is infinite for every $x \in A$.

THEOREM 9. Every weakly dimensionally complemented cylindric algebra is a diagonal cylindric algebra, and so is representable. ${ }^{8}$

Proof. Suppose $\mathfrak{U}$ is a $W D C A_{\alpha}, a \in A, a \neq 0$, and $F$ is a finite subset of $\alpha$. Choose $\kappa, \lambda$ distinct in $\alpha \sim F$ such that $c_{\kappa} c_{\lambda} a=a$. If $a \cdot d_{\kappa \lambda}=0$, then $a=0$, contradiction.

Theorem 10. Let $\alpha$ be an infinite ordinal, and let $\mathfrak{A}$ be a $C A_{\alpha}$. Suppose there is a finite subset $F$ of $\alpha^{2} \sim \delta_{\alpha}$ such that $\prod_{\langle\kappa, \lambda\rangle \in F}-d_{\kappa \lambda}=$ 0 . Then $\mathfrak{A}$ is a diagonal cylindric algebra, and so is representable. ${ }^{9}$

Proof. Suppose $a \in A, a \neq 0$, and $G$ is a finite subset of $\alpha$. Choose $\kappa \in \omega \sim 1$ and $\mu \in \alpha^{\kappa}$ such that $\mu$ maps $\kappa$ one-to-one onto the field of $F$, i.e., onto $\left\{\lambda \mid \mathrm{V}_{\nu<\alpha}(\langle\lambda, \nu\rangle \in F\right.$ or $\left.\langle\nu, \lambda\rangle \in F)\right\}$. Also choose $\nu \in \alpha^{\kappa}$ such that $\nu$ is one-to-one and range $\nu \subseteq \alpha \sim G \sim$ (field of $F$ ). Let

$$
H=\left\{\left\langle\nu \mu^{-1} \kappa, \nu \mu^{-1} \lambda\right\rangle \mid\langle\kappa, \lambda\rangle \in F\right\} .
$$

Applying $S_{\nu_{0}}^{\mu_{0}} \cdots S_{\nu_{\kappa-1}}^{\mu_{\kappa-1}}$ to $\prod_{\langle\kappa, \lambda\rangle \in F}-d_{\kappa \lambda}$, we see that $\Pi_{\langle\kappa, \lambda\rangle \epsilon_{H}}-d_{\kappa \lambda}=0$. Moreover, $H$ is a finite subset of $\alpha^{2} \sim \delta_{\alpha}$ such that (field of $H$ ) $\cap G=0$. Since $a \neq 0$, choose $\langle\kappa, \lambda\rangle \in H$ such that $a \cdot d_{\kappa \lambda} \neq 0$. Thus $\mathfrak{A}$ is a diagonal cylindric algebra.

In conclusion, we would like to make a few remarks about the general theory of diagonal cylindric algebras. In the first place, $G C A_{\alpha}$ is properly included in $R C A_{\alpha}$; the cylindric set algebra formed from all subsets of $\omega^{\omega}$ forms an example of an element of $R C A_{\alpha} \sim G C A_{\alpha}$; in this algebra the element $\left\{\delta_{\omega}\right\}$ is included in the complement of every nonunity diagonal element. Clearly $G C A_{\alpha}$ is closed under direct products and subalgebras. But from Theorem 2.19 of [5] it follows that $G C A_{\alpha}$ is not equational, and so is not closed under homomorphisms. For,

8 This is a solution of a problem of Henkin and Tarski, who showed that $\mathscr{U}$ is representable if $\alpha \sim(\Delta x \cup \Delta y)$ is infinite for all $x, y \in A$.

${ }^{9}$ Actually a somewhat stronger theorem holds. In fact, instead of assuming that $F$ is finite, it suffices to assume that $\alpha \sim$ Field $(F)$ is infinite. Then, in general, the product mentioned in Theorem 10 may be an infinite product. 
$L C A_{\alpha} \subseteq G C A_{\alpha} \subset R C A_{\alpha}$, and by the quoted theorem $R C A_{\alpha}$ is the smallest equational class including $L C A_{\alpha}$.

\section{BIBLIOGRAPHY}

1. Garrett Birkhoff, Subdirect unions in universal algebra, Bull. Amer. Math. Soc., 50 (1944), 764-768.

2. B. A. Galler, Cylindric and polyadic algebras, Proc. Amer. Math. Soc., 8 (1952), 176183.

3. Paul R. Halmos, Algebraic logic IV, Trans. Amer. Math. Soc., 86 (1957), 1-27.

4. Leon Henkin and Alfred Tarski, Cylindrical algebras, Summaries of talks presented at the Summer Institute of Symbolic Logic v. 3 Cornell 1957, 332-340.

5. - Cylindric algebras, Proc. of symposia in pure math. v. 2, Lattice Theory, 1960, Amer. Math. Soc.

6. H. J. Keisler, The representation of polyadic algebras of infinite degree, Notices Amer. Math. Soc., 7 (1960), 364 (Abstract 569-48).

7. John L. Kelley, General Topology, 1955.

8. Donald Monk, Non-representable polyadic algebras of finite degree, Notices Amer. Math. Soc., 7 (1960), 735 (Abstract 574-5).

9. Alfred Tarski, Contributions to the theory of models I, II III, Proc. Neder. Ak. van Weten. Ser. A 57: 572-591; 58: 56-64.

UNIVERSITY OF CALIFORNIA, BERKELEY 



\section{PACIFIC JOURNAL OF MATHEMATICS}

\section{EDITORS}

\author{
RaLPh S. Phillips \\ Stanford University \\ Stanford, California \\ F. H. BRowNELL \\ University of Washington \\ Seattle 5 , Washington
}

A. L. Whiteman

University of Southern California

Los Angeles 7, California

L. J. Paige

University of California

Los Angeles 24, California

\author{
E. F. BECKENBACH \\ T. M. CHERRY
}

\author{
ASSOCIATE EDITORS

$\begin{array}{lll}\text { D. DERRY } & \text { H. L. ROYDEN } & \text { E. G. STRAUS } \\ \text { M. OHTSUKA } & \text { E. SPANIER } & \text { F. WOLF }\end{array}$

\section{SUPPORTING INSTITUTIONS}

\author{
UNIVERSITY OF BRITISH COLUMBIA \\ CALIFORNIA INSTITUTE OF TECHNOLOGY \\ UNIVERSITY OF CALIFORNIA \\ MONTANA STATE UNIVERSITY \\ UNIVERSITY OF NEVADA \\ NEW MEXICO STATE UNIVERSITY \\ OREGON STATE COLLEGE \\ UNIVERSITY OF OREGON \\ OSAKA UNIVERSITY \\ UNIVERSITY OF SOUTHERN CALIFORNIA
}

\author{
STANFORD UNIVERSITY \\ UNIVERSITY OF TOKYO \\ UNIVERSITY OF UTAH \\ WASHINGTON STATE COLLEGE \\ UNIVERSITY OF WASHINGTON \\ AMERICAN MATHEMATICAL SOCIETY \\ CALIFORNIA RESEARCH CORPORATION \\ HUGHES AIRCRAFT COMPANY \\ SPACE TECHNOLOGY LABORATORIES \\ NAVAL ORDNANCE TEST STATION
}

Mathematical papers intended for publication in the Pacific Journal of Mathematics should be typewritten (double spaced), and the author should keep a complete copy. Manuscripts may be sent to any one of the four editors. All other communications to the editors should be addressed to the managing editor, L. J. Paige at the University of California, Los Angeles 24, California.

50 reprints per author of each article are furnished free of charge; additional copies may be obtained at cost in multiples of 50 .

The Pacific Journal of Mathematics is published quarterly, in March, June, September, and December. The price per volume (4 numbers) is $\$ 12.00$; single issues, $\$ 3.50$. Back numbers are available. Special price to individual faculty members of supporting institutions and to individual members of the American Mathematical Society: $\$ 4.00$ per volume; single issues, $\$ 1.25$.

Subscriptions, orders for back numbers, and changes of address should be sent to Pacific Journal of Mathematics, 103 Highland Boulevard, Berkeley 8, California.

Printed at Kokusai Bunken Insatsusha (International Academic Printing Co., Ltd.), No. 6, 2-chome, Fujimi-cho, Chiyoda-ku, Tokyo, Japan.

\section{PUBLISHED BY PACIFIC JOURNAL OF MATHEMATICS, A NON-PROFIT CORPORATION}

The Supporting Institutions listed above contribute to the cost of publication of this Journal, but they are not owners or publishers and have no responsibility for its content or policies.

Reprinted 1966 in the United States of America 


\section{Pacific Journal of Mathematics}

\section{Vol. 11, No. 4}

A. V. Balakrishnan, Prediction theory for Markoff processes . . . . . . . . . . 1171

Dallas O. Banks, Upper bounds for the eigenvalues of some vibrating systems . . . . 1183

A. Białynicki-Birula, On the field of rational functions of algebraic groups ...... 1205

Thomas Andrew Brown, Simple paths on convex polyhedra .............. 1211

L. Carlitz, Some congruences for the Bell polynomials . . . . . . . . . . . . 1215

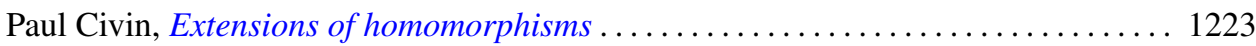

Paul Joseph Cohen and Milton Lees, Asymptotic decay of solutions of differential

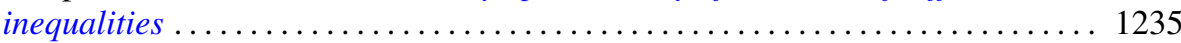

István Fáry, Self-intersection of a sphere on a complex quadric . . . . . . . . . . 1251

Walter Feit and John Griggs Thompson, Groups which have a faithful representation

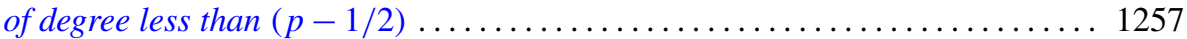

William James Firey, Mean cross-section measures of harmonic means of convex

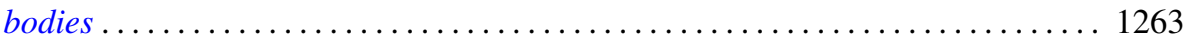

Avner Friedman, The wave equation for differential forms . . . . . . . . . . 1267

Bernard Russel Gelbaum and Jesus Gil De Lamadrid, Bases of tensor products of

Banach spaces ................................... 1281

Ronald Kay Getoor, Infinitely divisible probabilities on the hyperbolic plane . . . . 1287

Basil Gordon, Sequences in groups with distinct partial products . . . . . . . . . . . . 1309

Magnus R. Hestenes, Relative self-adjoint operators in Hilbert space . . . . . . . . . 1315

Fu Cheng Hsiang, On a theorem of Fejér ......................... 1359

John McCormick Irwin and Elbert A. Walker, On N-high subgroups of Abelian

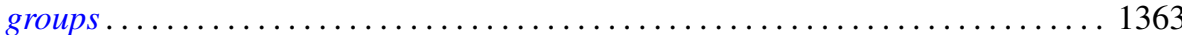

John McCormick Irwin, High subgroups of Abelian torsion groups . . . . . . . . . 1375

R. E. Johnson, Quotient rings of rings with zero singular ideal . . . . . . . . . . . 1385

David G. Kendall and John Leonard Mott, The asymptotic distribution of the time-to-escape for comets strongly bound to the solar system ...

Kurt Kreith, The spectrum of singular self-adjoint elliptic operators ....

Lionello Lombardi, The semicontinuity of the most general integral of the calculus of variations in non-parametric form ................................

Albert W. Marshall and Ingram Olkin, Game theoretic proof that Chebyshev inequalities are sharp

Wallace Smith Martindale, III, Primitive algebras with involution . . William H. Mills, Decomposition of holomorphs ..............

James Donald Monk, On the representation theory for cylindric algebras . . . . . . 1447

Shu-Teh Chen Moy, A note on generalizations of Shannon-McMillan theorem . . . . 1459

Donald Earl Myers, An imbedding space for Schwartz distributions . .

John R. Myhill, Category methods in recursion theory .........

Paul Adrian Nickel, On extremal properties for annular radial and circular slit mappings of bordered Riemann surfaces

Edward Scott O'Keefe, Primal clusters of two-element algebras . .

Nelson Onuchic, Applications of the topological method of Wazewski to certain

problems of asymptotic behavior in ordinary differential equations ...

Peter Perkins, A theorem on regular matrices................

Clinton M. Petty, Centroid surfaces .... 